${ }^{4}$ Goldberg, D., Genetic Algorithms in Search, Optimization and Machine Learning, Addison Wesley Longman, Reading, MA, 1989, p. 22.

${ }^{5}$ Isaacs, R., Differential Games, Wiley, New York, 1965, p. 279

${ }^{6}$ Horie, K., "Collocation with Nonlinear Programming for Two-Sided Flight Path Optimization," Ph.D. Dissertation, Dept. of Aeronautical and Astronautical Engineering, Univ. of Illinois at Urbana-Champaign, Urbana, IL, Feb. 2002.

${ }^{7}$ Goldberg, D. E., and Voessner, S., "Optimizing Global-Local Search Hybrids," Proceedings of the Genetic and Evolutionary Computation Conference 1999, Morgan Kaufmann, San Francisco, 1999.

\section{Circular Navigation Missile Guidance with Incomplete Information and Uncertain Autopilot Model}

\author{
Ian R. Manchester* and Andrey V. Savkin ${ }^{\dagger}$ \\ University of New South Wales, \\ Sydney, New South Wales 2052, Australia
}

\section{Introduction}

$\mathbf{T}$ HE vast majority of guidance laws have one objective: to reduce to zero the distance between the missile and the target (see Refs. 1 and 2). This is not always sufficient; in some cases the direction from which the missile approaches the target is also important. These include situations where a heavily armored target is best hit from a specific angle or when it is desired to disable a plane or vehicle without hitting either a dangerous payload or the pilot. This problem has been considered in a number of papers (for example, see Refs. 3-10). There are also connections between guidance problems of this sort and trajectory control of unmanned aerial vehicles and autonomous robots.

Circular navigation guidance (CNG) is a novel approach to this problem, first presented in Ref. 3. It is formulated for twodimensional planar intercepts and is built on a geometric principle that allows a relatively simple feedback control law to give probably perfect intercepts against nonmaneuvering targets.

The principle behind the control law is this: basic geometrical considerations provide us with an line-of-sight angle condition, which, if maintained, results in the missile following a circular path to the target and impacting with the desired approach angle. The job of maintaining this condition, when it is represented in appropriate variables, is that of regulating a linear system to a particular trajectory. This can then be solved with a feedforward control, which equals the control on the nominal path, and a proportional controller to regulate the missile to the nominal path.

The proof of perfect performance in Ref. 3 assumes that full state information (i.e., target location and velocity) is available, there is no delay in the autopilot, and the target is not maneuvering. (It has constant velocity.) In practice, none of these assumptions are strictly justifiable: it is usually impossible to have complete information about the state of the intercept, so that a state estimator, based on some mathematical system model, must be used. Also, in practice, there is an unavoidable delay between acceleration commands being given and being realized, that is, the autopilot is never perfect, and of course maneuvering targets must be taken into account.

Received 25 June 2003; revision received 21 April 2004; accepted for publication 7 May 2004. Copyright (C) 2004 by the American Institute of Aeronautics and Astronautics, Inc. All rights reserved. Copies of this paper may be made for personal or internal use, on condition that the copier pay the $\$ 10.00$ per-copy fee to the Copyright Clearance Center, Inc., 222 Rosewood Drive, Danvers, MA 01923; include the code 0731-5090/04 \$10.00 in correspondence with the CCC.

*Research Student, School of Electrical Engineering and Telecommunications; ianm@student.unsw.edu.au. Student Member AIAA.

${ }^{\dagger}$ Professor of Electrical Engineering.
In many target tracking applications where the underlying model is nonlinear (as it is in this case), an approximate generalization of the optimal linear Kalman filter to nonlinear dynamical systems, termed the extended Kalman filter (EKF) is used. However, this does not take into account uncertainty in the system model used. When such uncertainty is large enough, the estimated state has been shown to diverge from the true state values (see Ref. 11). Recent work drawing on robust control theory has provided a new approach to this problem. The robust extended Kalman filter (REKF-see Ref. 11 and see also Ref. 12 for the linear robust Kalman filter) increases the robustness of the filter to uncertainties satisfying a certain integral quadratic constraints. Like the EKF, the REKF is based on a linearization approach and hence cannot guarantee convergence of the estimate of a nonlinear system, but simulation studies have shown it is less susceptible to such problems.

The REKF is based on the framework of integral quadratic constraints, a description of uncertainty that has seen increasing attention of late in the robust control literature. ${ }^{11-14}$ This framework renders solvable robust control and estimation problems, which, under other frameworks, are not mathematically tractable.

In this Note we consider the case where only range and line-ofsight angle measurements are available (each corrupted by random noise); the autopilot is modeled by a second-order system with an uncertain parameters, and target maneuvers are modeled using a modified Singer method ${ }^{15}$ with uncertain bandwidth. The robust extended Kalman filter is used to estimate the state, and robust control techniques similar to $H^{\infty}$ methods are used to design the guidance law.

In the following four sections, we introduce CNG, extend it to an uncertain autopilot model, then design a robust state estimator, and finally test the complete system with computer simulations.

\section{Circular Navigation Guidance}

The circular navigation guidance law has two objectives: 1) minimize miss distance and 2) achieve impact from a specific angle, equal to the target's velocity vector plus some offset $\beta$. So let us say, referring to Fig. 1, that the missile should approach the target from a direction direction of the $Z$ vector immediately before impact. That is, the angle $\sigma$ should be equal to $\gamma_{T}+\beta$ immediately before impact.

The philosophy behind CNG is that two points, and a tangent on one of them, uniquely define a circle in the plane. Thus, a missile and target position, and a desired approach angle, uniquely define a circular path the missile can take to the target. An interesting geometric invariant was found that allows easy design of a feedback control law to keep a missile on such a path. For a more detailed discussion of this, see Ref. 3. In this Note we start with the definition of the control law and subsequently develop its extension to uncertain output feedback systems.

The control law is based on planar geometry, and so to formulate it in terms of a Cartesian state it is helpful to introduce some intermediate variables with some qualitative meaning. We now present the
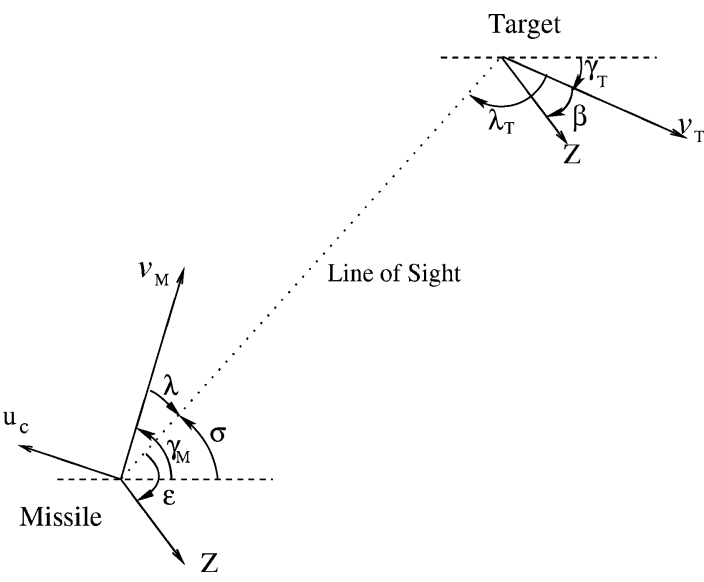

Fig. 1 Engagement geometry. 
idealized model in Cartesian form and from this derive the variables that are most useful in presenting the guidance law.

Firstly, we state some notation: Throughout the paper $I_{a}$ refers to the $a$-dimensional identity matrix, and $0_{a, b}$ refers to an $a \times b$ matrix of zeros.

We introduce three two-dimensional real vectors in Cartesian coordinates that together fully describe the state of the system: relative position $\boldsymbol{x}_{R}(t):=$ target position-missile position, missile velocity $\boldsymbol{v}_{M}(t)$, and target velocity $\boldsymbol{v}_{T}(t)$.

Now let a state vector $\boldsymbol{x}(t)$ represent these combined:

$$
\boldsymbol{x}(t):=\left[\begin{array}{c}
x_{1}(t) \\
\vdots \\
x_{6}(t)
\end{array}\right]:=\left[\begin{array}{c}
\boldsymbol{x}_{R}(t) \\
\boldsymbol{v}_{M}(t) \\
\boldsymbol{v}_{T}(t)
\end{array}\right] \in \mathbb{R}^{6}
$$

System dynamics are defined in terms of a nonlinear state-space model,

$$
\dot{\boldsymbol{x}}(t)=A \boldsymbol{x}(t)+B_{1}(\boldsymbol{x}) a_{M}(t)+B_{2}(\boldsymbol{x}) a_{T}(t)
$$

where $a_{M}(t)$ is the missile acceleration, which must be perpendicular to the missile's current velocity vector (i.e., the missile can perform turning motions). In Ref. 3 it was assumed we could assign this acceleration directly. Similarly, $a_{T}(t)$ is a one-dimensional acceleration issued perpendicular to the target's velocity, if the target is maneuvering. It might be thought of as the disturbance input.

Using a simple model of Newtonian physics, and the acceleration restrictions just described, we obtain the following definition of the matrices in Eq. (1):

$$
\begin{gathered}
A:=\left[\begin{array}{ccc}
0_{2,2} & -I_{2} & I_{2} \\
0_{4,2} & 0_{4,2} & 0_{4,2}
\end{array}\right] \\
B_{1}(\boldsymbol{x}):=\left[\begin{array}{c}
0_{2,1} \\
-x_{4}(t) / c_{M} \\
x_{3}(t) / c_{M} \\
0_{2,1}
\end{array}\right], \\
B_{2}(\boldsymbol{x}):=\left[\begin{array}{c}
0_{4,1} \\
-x_{6}(t) / c_{T} \\
x_{5}(t) / c_{T}
\end{array}\right]
\end{gathered}
$$

where

$$
\begin{aligned}
c_{M} & :=\sqrt{x_{3}(t)^{2}+x_{4}(t)^{2}} \\
C_{T} & :=\sqrt{x_{5}(t)^{2}+x_{6}(t)^{2}}
\end{aligned}
$$

are the missile and target speed, respectively.

Furthermore, we define the following variables, visualized in Fig. 1:

$$
\begin{gathered}
r(t):=\sqrt{x_{1}(t)^{2}+x_{2}(t)^{2}} \\
\sigma(t):=\tan ^{-1}\left[x_{2}(t) / x_{1}(t)\right] \\
\gamma_{M}(t):=\tan ^{-1}\left[x_{4}(t) / x_{3}(t)\right] \\
\gamma_{T}(t):=\tan ^{-1}\left[x_{6}(t) / x_{5}(t)\right] \\
\lambda(t):=\sigma(t)-\gamma_{M}(t) \\
\lambda_{T}(t):=\pi+\sigma(t)-\gamma_{T}(t) \\
\varepsilon(t):=\gamma_{T}(t)+\beta-\sigma(t) \\
\lambda_{\text {off }}:=-\sin ^{-1}\left[\left(c_{T} / c_{M}\right) \sin \lambda_{T}(t)\right]
\end{gathered}
$$

Note that, by $\tan ^{-1}(\boldsymbol{y} / \boldsymbol{x})$ we refer to the four-quadrant arctangent, which takes $\boldsymbol{x}$ and $\boldsymbol{y}$ as arguments and maps into the full circle $(-\pi, \pi]$. In MATLAB ${ }^{\circledR}$, for example, this is calculated with the ATAN2 command.

Qualitatively, $r(t)$ is the range between missile and target, $c_{M}$ is the speed of the missile, and $c_{T}$ the speed of the target. Note that
$c_{M}$ and $c_{T}$ are not time dependent: we are assuming that missile and target speeds are constant. The angle $\sigma(t)$ is that of the line of sight (LOS) between missile and target, $\gamma_{M}(t)$ is the missiles heading angle, and $\gamma_{T}(t)$ is the target's heading angle. All of the preceding angles are with respect to a horizontal ( $x_{1}$ direction) reference, where a positive angle is one of anticlockwise rotation.

We have also defined some relative angles of interest: $\lambda(t)$ is the angle between the line-of-sight vector and the missile's velocity vector, $\lambda_{T}(t)$ is the equivalent value for the target, and $\varepsilon(t)$ is the angle between the desired impact velocity vector and the line-ofsight vector (see Fig. 1). The reasoning for $\lambda_{\text {off }}$ is explained in Ref. 3 .

Let $k_{p}$ be some unitless gain term, then the control signal from ${ }^{3}$ $u_{c}(t)$ is calculated as follows:

$$
\begin{aligned}
u_{f}(t) & :=\frac{c_{M}}{r(t)}\left\{c_{M} \sin [\lambda(t)]+c_{T} \sin \left[\lambda_{T}(t)\right]\right\} \\
& \times\left\{2+\frac{\left(c_{T} / c_{M}\right) \cos \left[\lambda_{T}(t)\right]}{\sqrt{1-\left(c_{T} / c_{M}\right)^{2} \sin ^{2}\left[\lambda_{T}(t)\right]}}\right\} \\
u_{p}(t) & :=k_{p}\left[\lambda(t)-\varepsilon(t)-\lambda_{\mathrm{off}}(t)\right] \\
u_{c}(t) & :=u_{f}(t)+u_{p}(t)
\end{aligned}
$$

where $u_{f}$ is the feedforward term and $u_{p}$ is the linear regulation term. Only relative angles and vector magnitudes are used in this definition, and so the guidance law is independent of coordinate basis.

In Ref. 3, the control signal $u_{f}$ was mathematically proven to give a perfect intercept when the model is nominal, as just defined, and full state information is available, and the following conditions hold: 1) the target is nonmaneuvering (i.e., has constant velocity); 2) $\gamma_{M}(0)$ can be specified by the guidance law; 3) $c_{M} \geq \sqrt{ }\left(2 c_{T}\right)$; and $|\varepsilon(0)| \leq \pi / 2$. The first two conditions can almost be removed: impact errors resulting from these can, in theory, be made arbitrarily small by including $u_{p}(t)$ and increasing the gain $k_{p}$. Numerical simulations have confirmed that CNG works well against maneuvering targets provided that the gain is high enough.

In this Note, we relax the assumptions of nominal physical model and perfect measurements. To do so,we extend CNG using some recent advances in the theory of robust control and filtering theory.

\section{Robust Control Design}

In contrast to our early work, ${ }^{3}$ we no longer assume that the acceleration of the missile can be directly assigned. Instead, there is a second-order transfer function from control command to missile acceleration. Furthermore, the coefficients of this transfer function are not exactly known.

The transfer function has the following form:

$$
\frac{a_{M}(s)}{u_{c}(s)}=\frac{\left(b+\Delta_{2} b_{u}\right)}{s^{2}+\left(a+\Delta_{1} a_{u}\right) s+\left(b+\Delta_{2} b_{u}\right)}
$$

where $\Delta_{1}$ and $\Delta_{2}$ can range over the interval $[-1,1]$. Note that for all $\Delta_{1}, \Delta_{2}$ the transfer function has unity gain.

Because the nominal path is close to circular, the missile's acceleration will be almost constant when following it. Moreover, because the transfer function of the autopilot has unity gain at $s=0$, the feedforward component of the control law remains unchanged.

The task of regulating the missile to its nominal path has, however, been changed significantly. For this we use a game-type robust controller, derived in a way similar to $H^{\infty}$ control.

The aim is to regulate the LOS angle $\lambda$ to $\varepsilon+\lambda_{\text {off }}$, and the missile's acceleration $a_{M}$ to $u_{f}$, using the control input $u_{c}$. To this end, we define the error vector $\boldsymbol{x}_{a}$, in which we have added hats to certain variables to emphasize that, in a real system, these will be calculated from a state estimate and not pure values.

$$
\boldsymbol{x}_{a}(t)=\left[\begin{array}{c}
\hat{\lambda}(t)-\left[\hat{\varepsilon}(t)+\hat{\lambda}_{\mathrm{off}}(t)\right] \\
\hat{a}_{M}(t)-u_{f}(t) \\
\dot{\hat{a}}_{M}(t)-\dot{u}_{f}(t)
\end{array}\right]
$$


which should be regulated to zero. For small deviations about the desired trajectory, the error dynamics can be described by the following uncertain linear system:

$$
\dot{\boldsymbol{x}}_{a}(t)=\left(F+H_{2} \Delta C_{1}\right) \boldsymbol{x}_{a}(t)+\left(H_{1}+H_{2} \Delta D_{1}\right) u_{c}(t)
$$

where

$$
\begin{array}{ccc}
F=\left[\begin{array}{ccc}
0 & 1 / c_{M} & 0 \\
0 & 0 & 1 \\
0 & -b & -a
\end{array}\right], & H_{1}=\left[\begin{array}{l}
0 \\
0 \\
b
\end{array}\right], & H_{2}=\left[\begin{array}{ll}
0 & 0 \\
0 & 0 \\
1 & 1
\end{array}\right] \\
C_{1}=\left[\begin{array}{ccc}
0 & -b_{u} & 0 \\
0 & 0 & -a_{u}
\end{array}\right], & D_{1}=\left[\begin{array}{c}
-b_{u} \\
0
\end{array}\right], & \|\Delta\| \leq I_{2}
\end{array}
$$

Here $\|\cdot\|$ denotes the induced matrix norm.

Now we define a quadratic cost function

$$
J=\int_{0}^{\infty}\left[\boldsymbol{x}_{a}(t)^{\prime} Q_{1} \boldsymbol{x}_{a}(t)+u(t)^{\prime} R_{1} u(t)\right] \mathrm{d} t
$$

We now propose to use the guaranteed cost control method (see Ref. 13, Chapter 5), which means that we minimize an upper bound on the cost function (9) over all possible systems satisfying Eq. (7).

To this end, let $X$ be a solution of the following Riccati equation:

$$
\begin{aligned}
{[F-} & \left.H_{1}\left(\epsilon R_{1}+D_{1}^{\prime} D_{1}\right)^{-1} D_{1}^{\prime} C_{1}\right]^{\prime} X \\
& +X\left[F-H_{1}\left(\epsilon R_{1}+D_{1}^{\prime} D_{1}\right)^{-1} D_{1}^{\prime} C_{1}\right] \\
& +\epsilon X H_{2} H_{2}^{\prime} X-\epsilon X H_{1}\left(\epsilon R_{1}+D_{1}^{\prime} D_{1}\right)^{-1} H_{1}^{\prime} X \\
& +(1 / \epsilon) C_{1}^{\prime}\left[I-D_{1}\left(\epsilon R_{1}+D_{1}^{\prime} D_{1}\right)^{-1} D_{1}^{\prime}\right] C_{1}+Q_{1}=0
\end{aligned}
$$

where $\epsilon$ is chosen so as to minimizeTrace $(X)$. Commercial packages such as MATLAB include algorithms for solving Riccati equations, and it is straightforward to do a one-parameter search over $\epsilon$ for the solution with the smallest trace.

From this we form the gain matrix:

$$
K_{1}=-\left(\epsilon R_{1}+D_{1}^{\prime} D_{1}\right)^{-1}\left(\epsilon H_{1}^{\prime} X+D_{1}^{\prime} C_{1}\right)
$$

Then the following control law is optimal in the guaranteed cost sense:

$$
u_{p}(t)=K_{1} \boldsymbol{x}_{a}(t)
$$

As just noted, because the nominal path is nearly circular (and exactly circular for a stationary target) the function $u_{f}(t)$ is almost constant. Hence its derivative $\dot{u}_{f}(t)$ is very nearly zero. Because it is quite a complicated function, we therefore approximate it as zero, and the last row of $\boldsymbol{x}_{a}(t)$ becomes simply $\dot{\hat{a}}_{M}(t)$.

Note that if all of the uncertainty terms are zero $\left(H_{2}, C_{1}, D_{1}=0\right)$, then this control strategy reduces to the standard linear quadratic regulator. This linear controller is then combined with the nonlinear feedforward term $u_{f}(t)$ from Eq. (2):

$$
u_{c}=u_{p}(t)+u_{f}(t)
$$

\section{Robust Nonlinear Estimator Design}

The state of our system needs to be estimated, the dynamic model is uncertain, and the measurements are nonlinearly related to the states. We achieve this by constructing a robust extended Kalman filter, which gives state estimates of a nonlinear system subject to an integral quadratic constraint.

The filter sees measurements of $r$ and $\lambda$, each corrupted by noise, denoted $v$. That is, the sensor provides the measurements

$$
\boldsymbol{y}(t)=\left[\begin{array}{l}
r(t) \\
\lambda(t)
\end{array}\right]+\boldsymbol{v}(t)
$$

The noise on the angle measurements is the sum of three components: a constant fading-noise component; a receiver-noise component, which grows with range; and a glint-noise component, which grows as the missile nears the target. Specifically,

$$
\sigma_{\lambda}^{2}(r)=\sigma_{f}^{2}+\sigma_{\mathrm{rn}}^{2} r^{4}+\sigma_{g}^{2} / r^{2}
$$

The noise on the range measurements is relatively small, and we assume it has stationary covariance $\sigma_{r}^{2}$.

The target is assumed to be maneuvering, and this is represented using a modified Singer model. ${ }^{15}$ The difference between our method and the standard Singer method is that the bandwidth of the maneuver model is uncertain.

Let $p$ be the nominal bandwidth of the maneuvers, $k$ be a constant representing the degree of uncertainty, and $\boldsymbol{w}(t)$ be a white noise signal. Then the target acceleration $a_{T}(t)$ is modeled like so:

$$
\dot{a}_{T}(t)=-(p+\Delta k p) a_{T}(t)+p \boldsymbol{w}(t)
$$

This model can be represented as a nominal system, plus an uncertainty subject to an integral quadratic constraint (IQC):

$$
\begin{gathered}
\dot{a}_{T}(t)=-p a_{T}(t)+p\left[\boldsymbol{w}_{m}(t)+w_{u}(t)\right] \\
\boldsymbol{z}(t)=k a_{T}(t)
\end{gathered}
$$

where the noise input $w_{u}$ is subject to an IQC:

$$
\int_{0}^{s}\left\|w_{u}(t)\right\| \mathrm{d} t \leq \int_{0}^{s}\|z(t)\| \mathrm{d} t
$$

The system (15) corresponds to an instantaneous inequality between $\left\|w_{u}\right\|$ and $\|z\|$; however, inequalities of this type have not been found to lead to tractable estimator designs. The integral inequality (16) is a weaker condition, but leads to straightforward estimator equations. Furthermore, this uncertainty description allows for nonlinear and time-varying uncertainties.

For a moment we regard only the nominal case: $k=0$; afterwards, the model uncertainty comes back into consideration.

If the target accelerates randomly with a variance of $\sigma_{a}^{2}$ and a bandwidth $p$, then the variance of the white-noise input to the Singer model is $\sigma_{w}^{2}=2 \sigma_{a}^{2} / p$. The covariance matrices of the measurement noise and disturbance terms are then

$$
\Sigma_{v}=\left[\begin{array}{cc}
\sigma_{r}^{2} & 0 \\
0 & \sigma_{\lambda}^{2}(\hat{\boldsymbol{x}})
\end{array}\right], \quad \Sigma_{w}=\left[\begin{array}{cc}
\sigma_{w}^{2} & 0 \\
0 & \sigma_{w}^{2}
\end{array}\right]
$$

These noise inputs can be combined in an integral quadratic constraint, so that each noise input contributes an equal amount to the left-hand side of the inequality:

$$
\int_{0}^{s} \boldsymbol{w}_{m}(t)^{\prime} \Sigma_{w}^{-1} \boldsymbol{w}_{m}(t)+\boldsymbol{v}(t)^{\prime} \Sigma_{v}^{-1}(t) \boldsymbol{v}(t) \mathrm{d} t \leq d
$$

Because $\boldsymbol{w}_{m}$ and $\boldsymbol{v}$ are random, different values of $d$ will represent different level sets of a probability density function. A robust extended Kalman filter with this IQC would have exactly the same equations as a standard extended Kalman filter.

The key advantage of the IQC framework, however, is that allows us to combine uncertainties that come from exogenous sources (in our case, measurement noise and target maneuver) with those which are state dependent (maneuver-model uncertainty).

In reintroducing the model uncertainty, we again form weighting matrices so that each uncertainty signal contributes an equal amount to the left-hand side of the IQC. So to include the model uncertainty, we need to know relative sizes of $w_{u}$ and $\boldsymbol{w}_{m}$. Let $q=\sigma_{u}^{2} / \sigma_{w}^{2}$ be their ratio. This can be arrived at by experimental studies, or theoretical models. Then all of the uncertainty in our system can be included in a single integral quadratic constraint:

$$
\int_{0}^{s} \boldsymbol{w}(t)^{\prime} Q \boldsymbol{w}(t)+\boldsymbol{v}(t)^{\prime} R(t) \boldsymbol{v}(t) \mathrm{d} t \leq d+\alpha \int_{0}^{s} z(t)^{\prime} z(t) \mathrm{d} t
$$


where

$$
Q^{-1}=\Sigma_{w}(1+q), \quad R^{-1}=\Sigma_{v}
$$

and

$$
\alpha=1 / \sigma_{w}^{2}(1+q)
$$

We assume that the filter gets information on the missile velocity $\boldsymbol{v}_{M}$ from an inertial navigation system unit. It would be straightforward to account for errors on this signal, but in this study we assume they are slight enough to be neglected.

The following equations describe the complete dynamical model for the filter:

$$
\begin{gathered}
\dot{\boldsymbol{x}}(t)=A_{e} \boldsymbol{x}(t)+B_{v} \boldsymbol{v}_{M}(t)+B_{w} \boldsymbol{w}(t) \\
\boldsymbol{y}(t)=C[\boldsymbol{x}(t)]+\boldsymbol{v}(t) \\
\boldsymbol{z}(t)=K \boldsymbol{x}(t)
\end{gathered}
$$

where

$$
\begin{gathered}
A_{e}=\left[\begin{array}{ccc}
0_{2,2} & I_{2} & 0_{2,2} \\
0_{2,2} & 0_{2,2} & I_{2} \\
0_{2,2} & 0_{2,2} & -p I_{2}
\end{array}\right], \quad B_{v}=\left[\begin{array}{c}
-I_{2} \\
0_{2,2} \\
0_{2,2}
\end{array}\right] \\
B_{w}=\left[\begin{array}{c}
0_{2,2} \\
0_{2,2} \\
p I_{2}
\end{array}\right], \quad K=\left[\begin{array}{ll}
0_{2,4} & k I_{2}
\end{array}\right] \\
C(\boldsymbol{x})=\left[\begin{array}{c}
\sqrt{x_{1}^{2}+x_{2}^{2}} \\
\tan ^{-1}\left(x_{2} / x_{1}\right)
\end{array}\right]
\end{gathered}
$$

Then the robust extended Kalman filter for the systems (19-21), subject to the IQC (18), is given by the following equations, where time dependence has been omitted for the sake of brevity:

$$
\begin{aligned}
\hat{\boldsymbol{x}}(0) & =\boldsymbol{x}_{0} \\
\dot{\hat{\boldsymbol{x}}}= & A_{e} \hat{\boldsymbol{x}}+B_{v} \boldsymbol{v}_{M} \\
& +P\left\{\nabla_{x} C(\hat{\boldsymbol{x}})^{\prime} R(\hat{\boldsymbol{x}})[\boldsymbol{y}-C(\hat{\boldsymbol{x}})]+\alpha K^{\prime} K \hat{\boldsymbol{x}}\right\}
\end{aligned}
$$

where $P(t)$ is the solution to the matrix Riccati differential equation:

$$
\begin{aligned}
P(0) & =0_{6,6} \\
\dot{P}= & P A_{e}^{\prime}+A_{e} P+B_{w} Q^{-1} B_{w}^{\prime}+P\left[\alpha K^{\prime} K\right. \\
& \left.-\nabla_{x} C(\hat{\boldsymbol{x}})^{\prime} R(\hat{\boldsymbol{x}}) \nabla_{x} C(\hat{\boldsymbol{x}})\right] P
\end{aligned}
$$

\section{Simulation Results}

In this section we present the results of some computer simulations, chosen to test the performance of the guidance law (2), (12), and (13), based on the state estimate (23) and (23).

We assume that the missile goes blind upon coming within $10 \mathrm{~m}$ of the target and holds the control signal equal to what it was outside this ball.

\begin{tabular}{|c|c|}
\hline Variable & Value \\
\hline$x(0)$ & {$\left[\begin{array}{llllll}866 & 500 & 600 & 0 & -25 & 97\end{array}\right]^{\prime}$} \\
\hline$\beta$ & $-165 \mathrm{deg}$ \\
\hline$c_{M}$ & $600 \mathrm{~m} / \mathrm{s}$ \\
\hline$c_{T}$ & $100 \mathrm{~m} / \mathrm{s}$ \\
\hline$b$ & 400 \\
\hline$a$ & 32 \\
\hline$K_{1}$ & {$\left[\begin{array}{lll}26.5 & -0.158 & 0.0384\end{array}\right]$} \\
\hline$Q_{1}$ & $\operatorname{diag}\left[\begin{array}{lll}1 \mathrm{e} 5 & 1 \mathrm{e} 2 & 0\end{array}\right]$ \\
\hline$R_{1}$ & 1 \\
\hline
\end{tabular}

In these simulations, the miss distance is defined as the smallest range attained between missile and target, and the angle error is defined in terms of relative positions at the time $\tau$ when $r(\tau)=10 \mathrm{~m}$ : angle error $\varepsilon(\tau)$ (see Fig. 2).

The numerical values listed in Tables 1 and 2 were used in all the simulations except where otherwise noted.

The simulation results plotted in Fig. 3 show the performance of CNG over a wide range of desired impact angles. These ranged from $\beta=-160 \mathrm{deg}$ (nearly head on), through tail on ( $\beta=0)$, up to +160 deg. In all cases the missile started from a position behind the tail of the missile. The black bar chart shows the percentage of intercepts that also achieved miss distance less than $5 \mathrm{~m}$ and angle
Table 1 System variables

Table 2 Noise and uncertainty values

\begin{tabular}{lc}
\hline \hline Variable & Value \\
\hline$\sigma_{a}^{2}$ & $20 \mathrm{~m}^{2} \mathrm{~s}^{-4}$ \\
$\sigma_{f}^{2}$ & $0.1 \mathrm{rad}^{2}$ \\
$\sigma_{\mathrm{rn}}^{2}$ & $1 e-11 \mathrm{rad}^{2} / \mathrm{m}^{4}$ \\
$\sigma_{g}^{2}$ & $10 \mathrm{rad}^{2} \mathrm{~m}^{2}$ \\
$\sigma_{r}^{2}$ & $4 \mathrm{~m}^{2}$ \\
$a_{u}$ & 5 \\
$b_{u}$ & 10 \\
$q$ & $2.5 e-3$ \\
$p$ & $0.2 \mathrm{~s}^{-1}$ \\
$k$ & 0.5 \\
\hline \hline
\end{tabular}

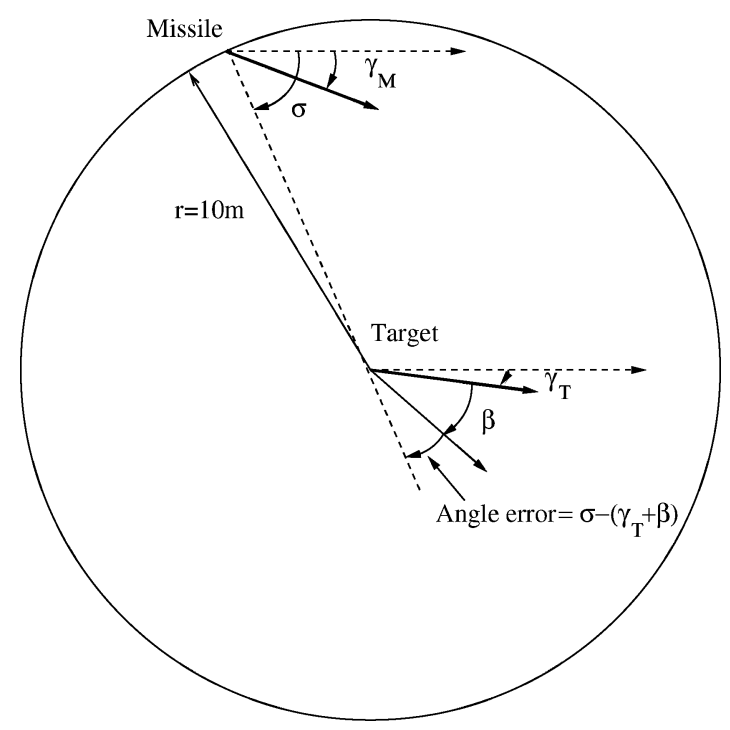

Fig. 2 Geometry for calculation of angle error.

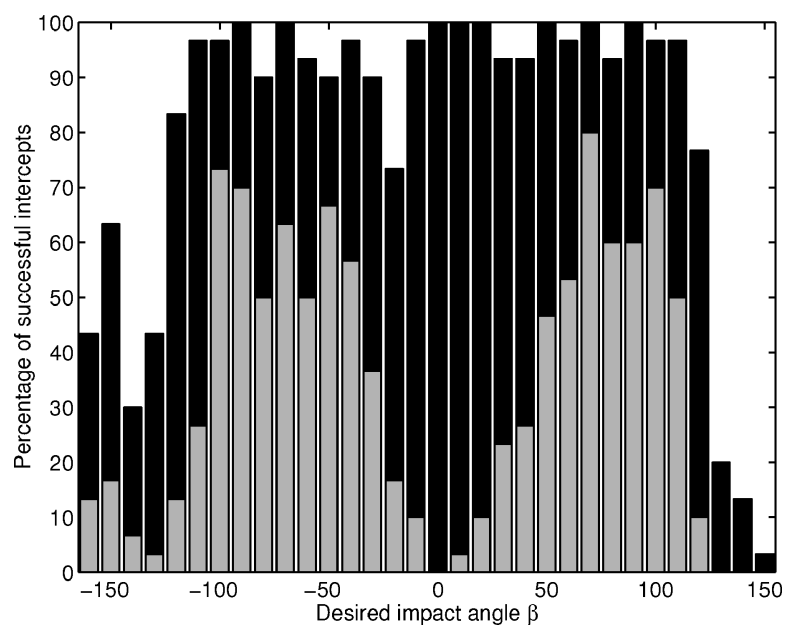

Fig. 3 Percentage of intercepts achieving angle error $5 \mathrm{deg}$, and miss distance $5 \mathrm{~m}$ (black) and $2 \mathrm{~m}$ (gray). 


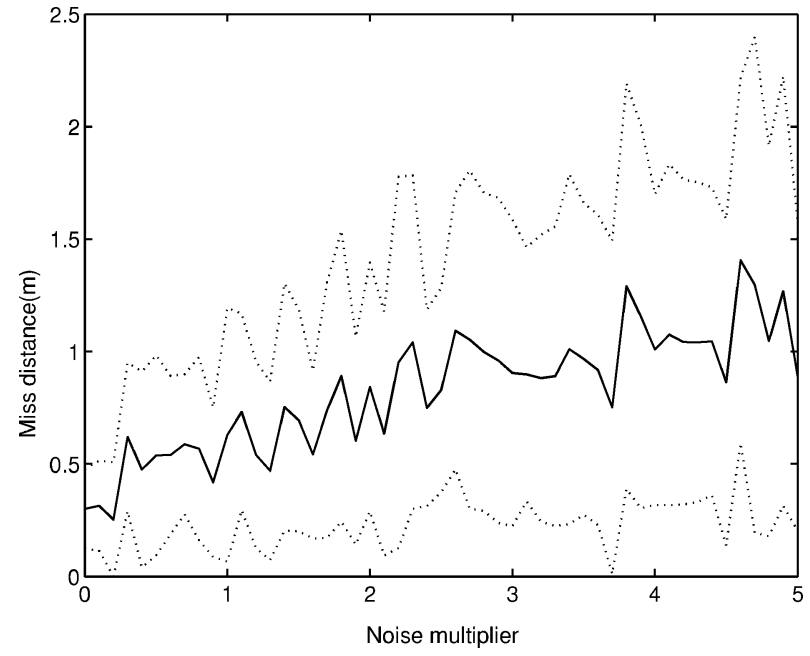

Fig. 4 Miss distance vs noise multiplier.

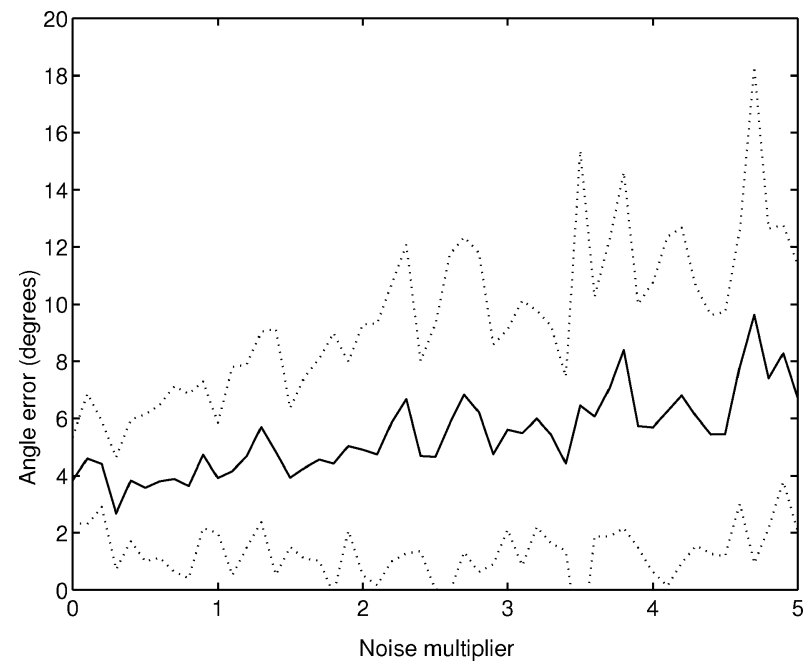

Fig. 5 Angle error vs noise multiplier.

error less than $5 \mathrm{deg}$. The gray bar chart in front shows those that also achieved a miss of less than $2 \mathrm{~m}$.

Good performance was achieved over nearly the full range of angles, but it is clear that the law performs particularly well when $\beta$ is around $\pm 90 \mathrm{deg}$, that is, when the missile is to hit the target from the side. We hypothesize that a reason for this is that the target maneuvers are orthogonal to its velocity, so that the missile sees them multiplied by the cosine of the approach angle.

We now take a more detailed look at a particularly difficult impact angle, $\beta=-165$. The initial conditions are as in Table 1 .

In Fig. 4 and 5 are the miss distances and angle errors over a range of measurement noise levels. That is, all values were as in Table 2, except the noise covariances $\sigma_{f}^{2}, \sigma_{\mathrm{rn}}^{2}, \sigma_{g}^{2}, \sigma_{r}^{2}$ were scaled by a factor between zero and five. As can be seen, both angle error and miss distance increase mildly as does the noise multiplier. The dotted lines represent standard deviations, which also increase mildly.

Similar plots are shown in Figs. 6 and 7, in which it was the maneuver covariance $\sigma_{a}^{2}$ from Table 2 that was scaled by factor varied from zero to five. Again, both the means and covariances increase mildly with the scaling factor. Of course, the relative susceptibilities of the filter to maneuvers, and to measurement noise, can be traded against one another by tuning the relevant covariance matrices.

The same studies were performed for various autopilot models with the bounds prescribed. It was found that there was very little difference. The reason for this is that, after an initial transient to reach the nominal circular path, the missile's acceleration is essentially constant, and so autopilot-model uncertainty has little effect. If the

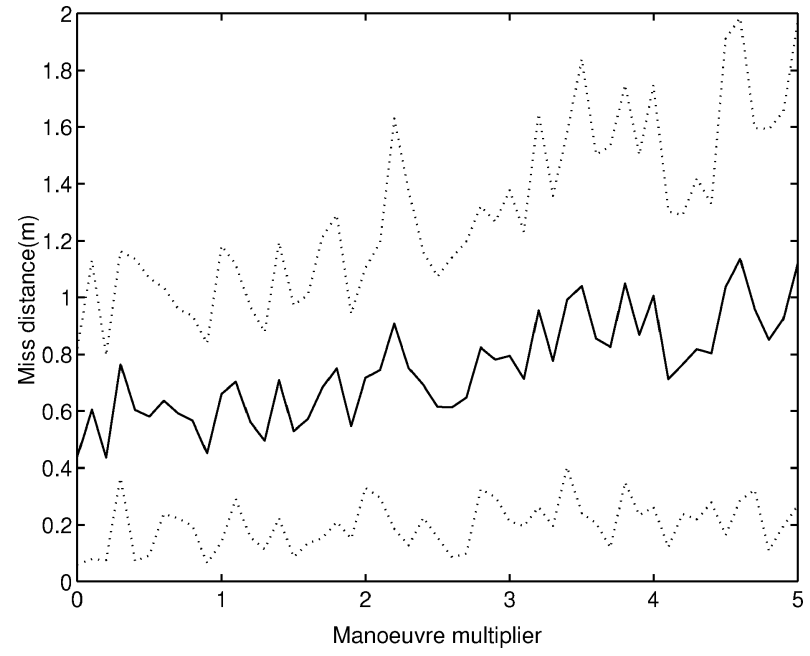

Fig. 6 Miss distance vs maneuver multiplier.

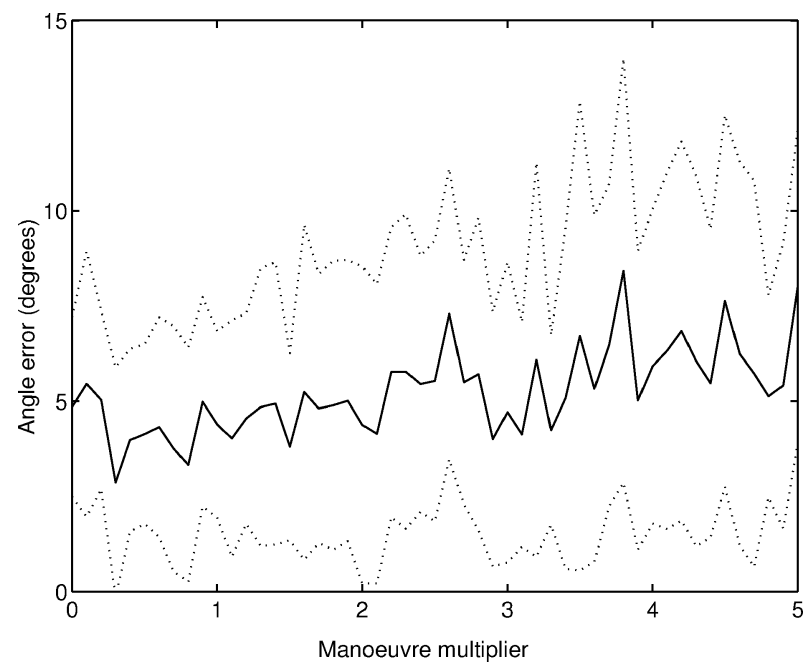

Fig. 7 Angle error vs maneuver multiplier.

range of possible autopilots included systems with nonunity gain, a greater effect would be seen.

\section{Acknowledgment}

This work was supported by the Australia Research Council.

\section{References}

${ }^{1}$ Zarchan, P., Tactical and Strategic Missile Guidance, AIAA, Washington DC, 1994

${ }^{2}$ Ben-Asher, J. Z., and Yaesh, I., Advances in Missile Guidance Theory, AIAA, Reston, VA, 1998.

${ }^{3}$ Manchester, I. R., and Savkin, A. V., "Circular Navigation Guidance Law for Precision Missile Target Engagements," Proceedings of the 41st IEEE Conference on Decision and Control, Vol. 2, Inst. of Electrical and Electronics Engineers, Piscataway, NJ, 2002, pp. 1287-1292.

${ }^{4}$ Manchester, I. R., Savkin, A. V., and Faruqi, F. A., "Optical-Flow Based Precision Missile Guidance Inspired by Honeybee Navigation," Proceedings of the 41st IEEE Conference on Decision and Control, Vol. 5, Inst. of Electrical and Electronics Engineers, Piscataway, NJ, 2003, pp. 5444-5449.

${ }^{5}$ Manchester, I. R., and Savkin, A. V., "Circular Navigation Missile Guidance with Incomplete Information and Uncertain Autopilot Model," AIAA Paper 2003-5448, Aug. 2003.

${ }^{6}$ Kim, M., and Grider, K. V., "Terminal Guidance for Impact Attitude Angle Constrained Flight Trajectories," IEEE Transactions on Aerospace and Electronic Systems, Vol. 9, No. 6, 1973, pp. 852-859.

${ }^{7}$ Kim, B. S., Lee, G. L., and Han, H. S., "Biased PNG Law for Impact with Angular Constraint," IEEE Transactions on Aerospace and Electronic Systems, Vol. 34, No. 1, 1998, pp. 277-288. 
${ }^{8}$ Song, T. L., Shin, J. S., and Han, H. S., "Impact Angle Control for Planar Engagements," IEEE Transactions on Aerospace and Electronic Systems, Vol. 35, No. 4, 1999, pp. 1439-1444.

${ }^{9}$ Song, T. L., and Shin, J. S., "Time Optimal Impact Angle Control for Vertical Plane Engagements," IEEE Transactions on Aerospace and Electronic Systems, Vol. 35, No. 2, 1999, pp. 738-742.

${ }^{10}$ Savkin, A. V., Pathirana, P., and Faruqi, F. A., "The Problem of Precision Missile Guidance: LQR and $\mathcal{H}_{\infty}$ Frameworks," IEEE Transactions on Aerospace and Electronic Systems, Vol. 39, No. 3, 2003, pp. 901-910.

${ }^{11}$ Petersen, I. R., and Savkin, A. V., Robust Kalman Filtering for Signals and Systems with Large Uncertainties, Birkhauser, Boston, MA, 1999.

${ }^{12}$ Savkin, A. V., and Petersen, I. R., "Recursive State Estimation for Uncertain Systems with an Integral Quadratic Constraint," IEEE Transactions on Automatic Control, Vol. 40, No. 6, 1995, pp. 1080-1083.

${ }^{13}$ Petersen, I. R., Ugrinovskii, V. A., and Savkin, A. V., Robust Control Design Using $H^{\infty}$ Methods, Springer-Verlag, London, 2000, Chap. 5.

${ }^{14}$ Savkin, A. V., and Petersen, I. R., "Nonlinear Versus Linear Control in the Absolute Stabilizability of Uncertain Linear Systems with Structured Uncertainty," IEEE Transactions on Automatic Control, Vol. 40, No. 1, 1995, pp. $122-127$.

${ }^{15}$ Singer, R. A., "Estimating Optimal Tracking Filter Performance for Manned Maneuvering Targets," IEEE Transactions on Aerospace and Electronic Systems, Vol. 6, No. 4, 1970, pp. 473-483.

\section{Optimal Dual-Rate Digital Redesign with Application to Missile Control}

\author{
C. A. Rabbath* \\ Defence Research and Development Canada-Valcartier, \\ Val-Belair, Quebec G3J 1X5, Canada \\ N. Lechevin \\ Université du Québec à Trois-Rivières, \\ Trois-Rivières, Quebec G9A 5H7, Canada \\ and \\ N. Hori \\ University of Tsukuba, Tsukuba 305-8573, Japan
}

\section{Introduction}

D IGITAL flight-control systems can be obtained via digital redesign; that is, a known continuous-time (CT) autopilot is converted to discrete time (DT) for digital implementation. With digital redesign, the DT controllers are obtained either by discretizing the individual CT controllers ${ }^{1}$ or by using a sophisticated method that takes into account the closed-loop topology and the dynamics of the system under control. ${ }^{2}$ The availability of a CT control system prior to the selection of the sampling rate for digital control is a key feature of digital redesign. However, the selection of the sampling rates is constrained by the hardware selected for control, sensing, and actuation. ${ }^{3}$ With single-rate DT systems, a high sampling rate is usually needed to guarantee closed-loop stability and performance, although, in practice, it may be inappropriate due to

Received 10 November 2003; revision received 6 May 2004; accepted for publication 3 June 2004. Copyright (C) 2004 by the authors. Published by the American Institute of Aeronautics and Astronautics, Inc., with permission. Copies of this paper may be made for personal or internal use, on condition that the copier pay the $\$ 10.00$ per-copy fee to the Copyright Clearance Center, Inc., 222 Rosewood Drive, Danvers, MA 01923; include the code 0731-5090/04 \$10.00 in correspondence with the CCC.

*Defence Scientist, 2459 Pie-XI N.; also Adjunct Professor, Department of Mechanical Engineering, McGill University, Montreal, Quebec H3A 2K6, Canada.

Adjunct Professor, Department of Electrical and Computer Engineering.

${ }^{\dagger}$ Professor, Graduate School of Systems and Information Engineering, Intelligent Interaction Technology Division, 1-1-1 Tennoudai. Member AIAA. the risk of numerical errors and the unavailability of converters of sufficiently high resolution and computers of appropriate processing power, especially when design constraints on size, mass, and power consumption are present. Multirate sampling can be used to optimize the allocation of processing power and to allow greater flexibility in the design of multichannel, multiloop autopilots. Using plant-output sampling and control-input update at different rates can provide better trade offs between performance and implementation costs; for instance, a dual-rate control synthesis and implementation scheme makes it possible to effectively handle multiple dynamic scales and constraints on hardware effectively. ${ }^{4}$

This note proposes an optimal dual-rate digital-redesign method that can be applied to CT and high-rate DT control systems. Briefly, given the existence of a CT control system, or of a fast DT control system, a DT $H_{2}$ optimal-control problem is solved to convert the known control system to either a low-rate or a dual-rate digital control system in a way that guarantees closed-loop stability and performance in the DT $\mathrm{H}_{2}$ sense. The idea of performing digital redesign using the $\mathrm{H}_{2}$ method on the closed-loop system comes from Ref. 5, although the methods presented there yield single-rate digital control systems. In this Note, the proposed dual-rate digital-redesign technique results in digital control systems that have satisfactory closedloop performance over an extended range of sampling rates, as compared with other widely used methods of digital redesign. Interestingly, the proposed dual-rate digital redesign applies to single- and multiloop systems. Furthermore, the proposed method of digital redesign makes use of generalized holds and samplers of the dual-rate type, thereby providing an added level of flexibility. ${ }^{6}$ The proposed dual-rate digital-redesign technique is, however, constrained by the specific requirements that arise when a DT $H_{2}$ problem is solved.

\section{Optimal Dual-Rate Digital Redesign}

\section{A. Assumptions}

Assumption 1: The uniform sampling periods are $h$ (low rate of $1 / h \mathrm{~Hz}$ ) and $T$ (high rate of $1 / T \mathrm{~Hz}$ ). The periods are related as follows: $h=N \cdot T, N \in Z^{+}$, where $Z^{+}$is the set of positive integers. $T$ is chosen to be nonpathological ${ }^{7}$ with respect to the plant transfer function.

Assumption 2: The hold device $H$ and ideal sampler $S$, which can each take a period equal to $T$ or $h$, are synchronized at time $t=0$. The hold has a bounded response to a unit DT impulse input and does not introduce any discrete zero into the hold-equivalent model of the plant, which cancels a pole of the plant model at nonpathological $T$ values. ${ }^{8}$ For example, the zero-order hold $(\mathrm{ZOH})$ satisfies this condition.

Assumption 3: Dual-rate digital redesign is performed with the objectives of preserving the step-input tracking performance ${ }^{6}$ and the step-disturbance rejection property of the CT (or fast DT) closedloop system.

\section{B. Proposed Method}

1. Step 1: Fast Discretization of CT Control System

Suppose that the control system to be redesigned is as shown in Fig. 1a. The CT control system provides satisfactory closed-loop performance. The CT plant $\bar{G}(s)$ may comprise actuator and sensor dynamics. Precede $\bar{G}(\underline{s})$ by a hold device $H$ and place the ideal sampler $S$ at the output of $\bar{G}(s)$. $H$ and $S$ are synchronized at the high rate of $1 / T$. The transfer function of $S \bar{G} H$ is given by $G(z, T)$. Proceed similarly for controller $\bar{C}(s)$. Then there results a fast, single-rate

a)
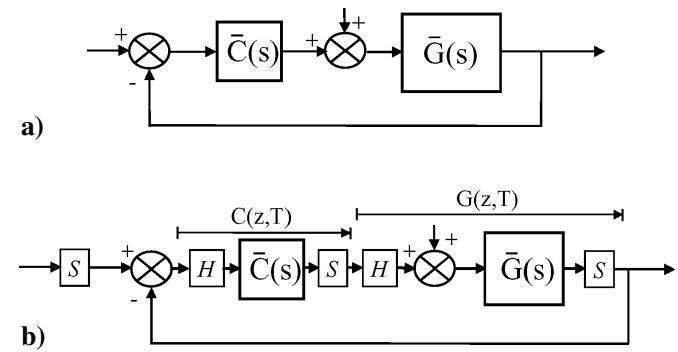

Fig. 1 Control system: a) CT and b) DT obtained by fast discretization. 